\title{
AI Based Traffic and Automobile Monitoring System
}

\author{
Sarika Chaudhary, Pooja Nagpal, Yomesh Devgan
}

\begin{abstract}
We live in a world where technology is constantly changing and upgrading, so this change gave birth to artificial intelligence (AI). When intelligence is shown by humans it's called human intelligence, but when machines show such intelligence it's called artificial intelligence. And by this constant change in technology we have opened doors to some new threats and humans are incapable to measure and predict such dangers. But on the other hand, AI in today's era has reached such potential that it can be used to monitor and predict many dangers. This paper presents a technique about how we can use artificial intelligence to monitor traffic in highways, roads streets and in many places where we cannot put traffic signals, and how we can monitor activity of each and every individual vehicle to make world safer for humans and other life forms so we can live in harmony and unison.
\end{abstract}

KEYWORDS- Cameras, GPS, 5G, machine learning, OS, object tracking.

\section{INTRODUCTION}

In very easy language we can say that any task performed by a human requires intelligence and it can be termed as human intelligence, and on the other hand when a task is performed by a machine which requires intelligence it uses artificial intelligence. AI is a very powerful tool only if humans can harness it properly, and how one can use features of AI to monitor the things which are difficult to measure due to human errors. When it comes to monitoring and keeping track, human beings are usually lethargic, less focused and can't multitask efficiently. But AI overcomes all these drawbacks and on a positive side AI doesn't get tired. Traffic and automobile monitoring System using A.I uses multiple technologies which can be unified for get out project up and running. We already have most of the technology needed at our disposal to do this task at a small scale.

\section{Manuscript received May 2, 2020}

Sarika Chaudhary, Assistant Professor, Department of Computer Science, Amity University, Gurugram, India, (e-mail: schaudhary@ggn.amity.edu).

Pooja Nagpal, Assistant Professor, Department of Computer Science, Amity University, Gurugram, India.

Yomesh Devgan, Department of Computer Science, Amity University, Gurgaon, Haryana, India.
But when we think of places where this system can be implemented are Dubai, America, japan and other major countries who fund in technology [4] and surveillance. In large cities, transport is becoming a growing challenge, both for commuters and for city officials who want to improve efficiency, reduce pollution and reduce frustration for drivers and commuters which will definitely lead to decrease in road accidents. We are in a condition where vehicles have become necessary evil, i.e., we need vehicles for nearly everything. And this need and overuse is creating a whole lot of problems which are now getting out of our hands to control manually.

A much-ignored fact is that an average person spends about 42 hours getting to and from work every year. In congested city areas that number skyrockets to even higher [1]. And when we do some math's we can come up to a number that on an average a person works for about 20-25 years and for every year we waste 50 hours of time so a number will be $1000-1250$ hours and that is a very huge number which is just wasted.

\section{RELATED TECHNOLOGIES}

Many countries are already working on AI so that it can monitor roads, streets and highways with the help of machine learning. By this technology we will be able to eliminate the need of posting policemen and prevent them from getting respiratory problem due to pollution. Which technologies will be a basic need for AI monitoring system of traffic and automobiles?

\section{A. A.I. Based Cameras}

Cameras will be needed which is a basic need of any traffic monitoring system[10], but with AI based computer vision cameras we will be able to track vehicles with much more efficiency than that of current system present anywhere. Motorized cameras with infrared which are controlled manually shall be replaces with machine learning cameras which will automatically work according to situation. Approximately, 7,500 cameras with multidirectional infrared and colorless laser sensors will count the volume on all arterial roads based on image pattern analysis, with image recognition [1].

\section{B. $5 G$}

$5 \mathrm{~g}$ is an upcoming technology which is a necessary for our model. We will be required to send and receive a lot of data for real time working of all the data. Greater throughput as range is from $50 \mathrm{mbps}$ to $10 \mathrm{gbps}$ [8]. Lower latency down to 1 millisecond and 5g have more connection density. 


\section{GPS}

GPS is another key aspect of this model, GPS is required to get real time data of traffic which will be inbuilt within every car and this data will be uploaded to the central database[11] unit from were cameras and traffic lights will work with syncing. GPS will also help in finding the stolen vehicles. Google maps is a prime example of GPS using app in real time. We can see all the detail of roads and traffic levels in real time, we can use this model of Google maps with AI based monitoring system [12].

\section{OS, Control Unit and Data Center}

A specific OS is required that is compatible with VPU (vision processing unit). VPU [6] is a segment of microprocessors which is specifically designed to work with AI. OS needs to robust and secure which can handle a lot of abuse without crashing or losing any data, which will obviously create problems, and the OS should be future proof which can also be able to connect to smart cars, this will be very helpful $\mathrm{n}$ betterment and increasing the efficiency of the system.

A control unit will be required where all the data will be stored safely, and no other person can access that data. In newer cars black box is being installed it can store all the data of any failure or accident of the vehicle. IO box will collect data analyze and use that data according to whatever the need is by using machine learning data processed will be used by AI.

\section{PROPOSED MODEL}

Many companies big like Mercedes-Benz, Google, Tesla, Audi, Volkswagen and Microsoft are already working on this project for the betterment of the society this artificial intelligence build system for monitoring traffic and vehicles.

On an average day more than 400 people die due to accident only in India on a single day. And the main reason of death is that they do not reason hospital on time, just because someone didn't report the accident [7] to the nearby authorities. So, we need to develop a monitoring system that aimed to save lives either by giving alternative routes to prevent getting stuck in heavy traffic areas, or in case of emergency contacting the nearby authorities automatically so that life can be saved. Various key factors considered for system monitoring are:

- Traffic management system

- Vehicle control and safety system

- Distinguishing between private and commercial vehicle[9]

- Distinguishing between cycle and motorcycle

Fig. 1 shows the blueprint of the model proposed and Fig. 2 represents the conceptual view of the proposed model.

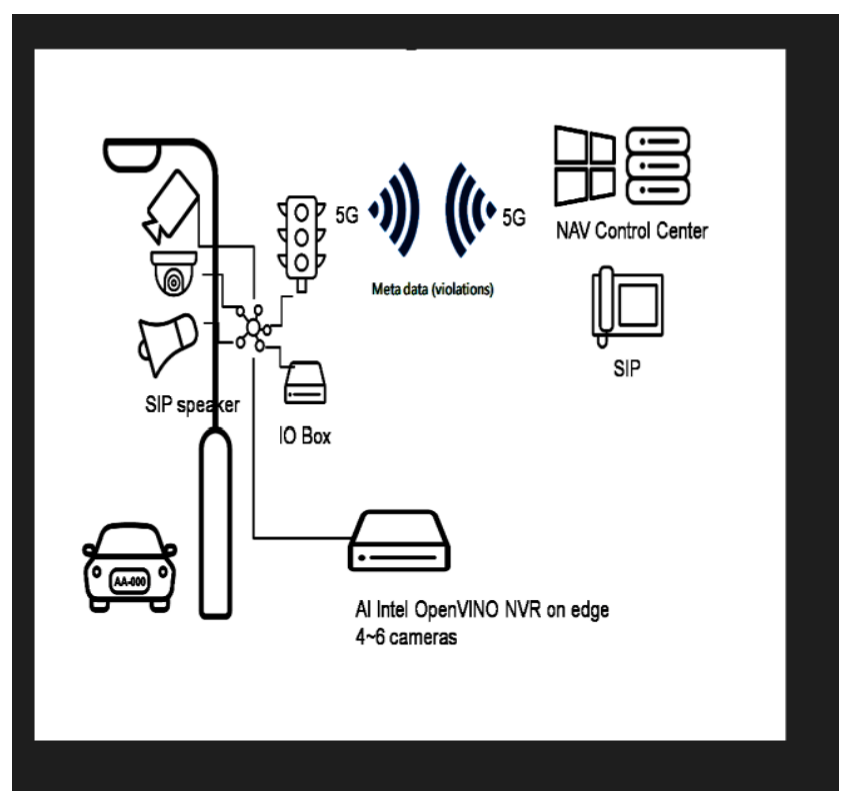

Fig.1: Proposed Model

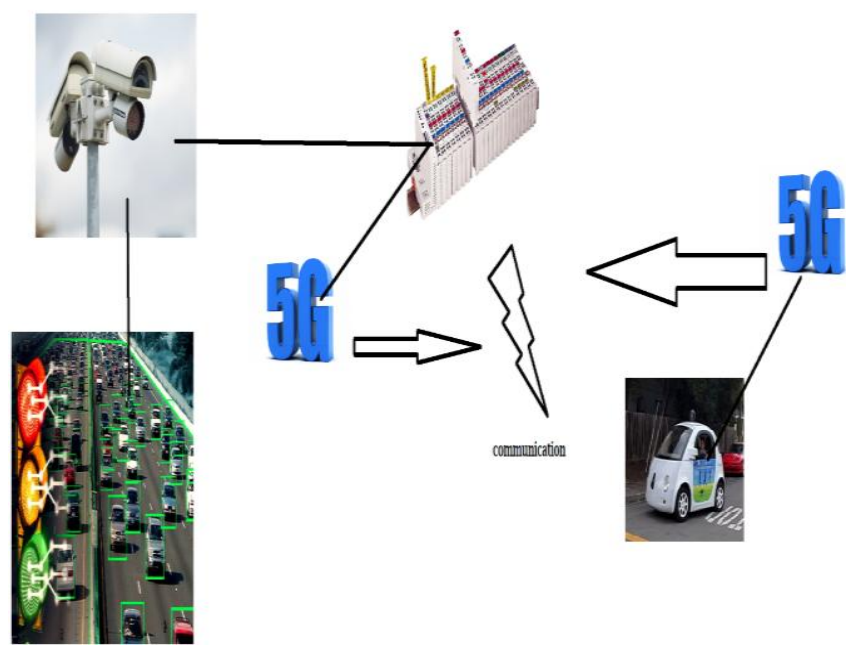

Fig.2: Conceptual view of Model

The working of proposed model is entirely based upon the AI, machine learning and 5G [3]. All of them are working together in Unison to achieve the desired goals of better traffic management and vehicle management system. $5 \mathrm{G}$ is used because of the minimum latency and it is the fastest technology by which we can use internet remotely. AI based cameras are to be used in this project for the best output and tracking of the subject. SIP is session initiation protocol, which is used for initiating, maintaining and terminating of the real time session that can be anything in form of voice, message or a video. Hence SIP speakers are to be used, SIP speakers are Power over Ethernet(PoE) and uses VoIP protocol which makes them widely used speaker for public addressing loudspeaker they are connected by CAT5 cable for high speed connection and nose resistant transmission .The access one can have after completion of this system is immense for example it can track a stolen vehicle and over speeding car or bike, by the power of AI the system can also contact the nearby authorities like hospital or police station in case of need if the vehicle has met with an accident. The system will notify the nearby authorities about the situation and this will help in saving lives immensely. The efficiency 
and versatility of the system is endless, all we need is to find the solution of the problems rather than procrastinating them. Fig.3. shows the steps of proposed methodology.

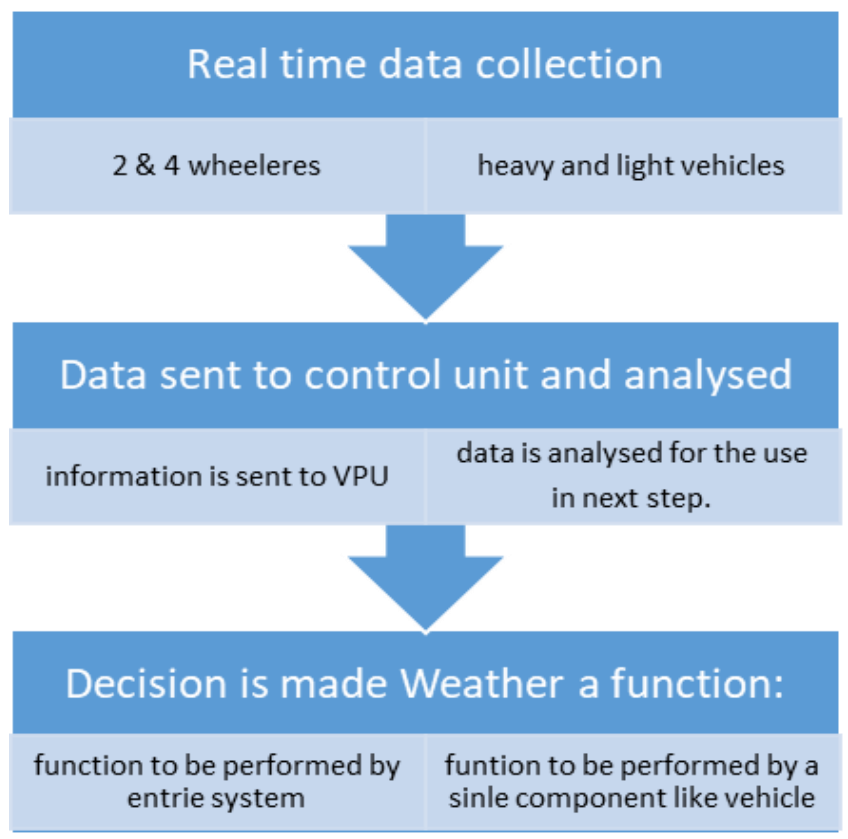

Fig.3: Steps in Proposed Methodology

The steps of implementation are explained below:

\section{A. Traffic data collection}

By using machine learning and collecting big data an Internet of Things [12] we will be able to collect real-time data off traffic. Some devices that will be used at GPS, road cameras and vehicle identifiers.

\section{B. Data transmission}

Data transmission is a crucial process in this system. As all the data collected will be sent to the control unit for processing by the VPU. Google already uses the information from phone's GPS which will be used by the system also.

\section{Traffic data analysis}

After all the data is sent and received now this is time to use that data by processing it and using it. The data is pooled so that appropriate conclusions and predictions can be made by $\mathrm{AI}$ and then send to the user that can be smart cars or phones [2].

\section{Traveler information and analysis}

Travel information will be the data which will be available to the user that can be a citizen or a smart car or a phone whatever the means necessary. It will be accessed via internet or other resources, which will later be used by the device registered for the use of the provided information. After all the data is collected, transmitted and analyzed then comes the role of Artificial intelligence to use and take actions with that knowledge. If the vehicle is travelling over the speed limit, then system sends a warning to the vehicle and the user, after 20 seconds it will issue an e-challan[5] on the name of the registered vehicle, and this system already is being implemented in many countries. Google on the other hand is trying to create a self-driven vehicle which can also be called a smart car. And with this technology fully created AI based monitoring system will become very efficient.

\section{CONCLUSION}

In this paper, we have examined many different permutations and combinations using AI to monitor traffic and automobile along with machine learning, to develop a system which is adaptive in real time situations. We have identified and compared many advantages and disadvantages in different situations. The proposed system has the capacity to provide us better traffic management system with minimum human intervention, least congestion of traffic, low pollution of air due to harmful fumes by decreasing the time of running of a vehicle on road, helping in emergencies where humans overall will lack, providing and creating a path to deal with emergency situation much more efficiently. In future we can predict an accident and counter it in real time, which will be possible in level 5 of automation of smart cars. Tesla the biggest electric vehicle company is on level 3, and their system has already shown evidences about predicting accidents even at level 3.

\section{REFERENCES}

[1] A. Jadhav, T.Ladhe, Kr.Yeolekar "Automated Attendance System Using Face Recognition" Irjet Volume: 04 Issue: 01, Jan -2017.

[2] L.Masupha, T. Zuva, S Ngwira, O.Esan "Face Recognition Techniques, Their Advantages, Disadvantages and Performance Evaluation" 2015 International Conference on Computing, Communication and Security (Icccs), 1-5,2015.

[3] Viani, F.; Robol, F.; Polo, A.; Rocca, P.; Oliveri, G.; Massa, A. Wireless architectures for heterogeneous sensing in smart home applications: Concepts and real implementation. Proc. IEEE 2013, 101, 2381-2396.

[4] Sapienza, M.; Guardo, E.; Cavallo, M.; La Torre, G.; Leombruno, G.; Tomarchio, O. Solving Critical Events through Mobile Edge Computing: An Approach for Smart Cities. In Proceedings of the 2016 IEEE International Conference on Smart Computing (SMARTCOMP), St. Louis, MO, USA, 18-20 May 2016; pp. 1729-1737.

[5] Bharti, M.; Saxena, S.; Kumar, R. Intelligent Resource Inquisition Framework on Internet-of-Things. Comput. Electr. Eng. 2017, 58, 265-281.

[6] Karan A Shah, Jasmine Jha2,"Improvement of Traffic Monitoring System by Density and Flow Control for Indian Road System Using IoT ".

[7] Sonali P. Kshirsagar, Priyanka H. Mantala, Gayatri D. Parjane, Kalyani G. Teke, "Intelligent Traffic Management based on IoT", International Journal of Computer Applications (0975 - 8887) 2017.

[8] V.M Baskaran, S.K Tiong, M.Z Jamaludin, "Analysis of Real-Time Multiple Source Video 
Streaming via Wireless $5.8 \mathrm{GHz}$ for Intelligent Traffic Management System" ,IEEE 2005.

[9] Megha C. Narhe, Dr. M. S. Nagmode ,"Vehicle Counting using Video Image Processing ",IJCAT 2014.

[10] M Febrian Rachmadi, Faris Al Afif, Wisnu Jatmiko, Petrus Mursanto, Manggala E A, and M Anwar Ma'sum, "Adaptive Traffic Signal Control System Using Camera Sensor and Embedded System", IEEE (2011).

[11] K.Ranjin,A.Kanthimathi Y.Yasmine ,"Design of Adaptive Road Traffic Control System through Unified Modeling Language",IEEE 2017.

[12] S.Chaudhary, P.B.Nagpal, "Live location tracker", Global Research and Development Journal for Engineering | Vol. 4 Issue.10,2019. 